\title{
EAR BASED HUMAN IDENTIFICATION USING A COMBINATION OF WAVELETS AND MULTI-SCALE LOCAL BINARY PATTERN
}

\author{
Pallavi Srivastava $^{1 *}$, Diwakar Agarwal ${ }^{2}$ and Atul Bansal ${ }^{3}$ \\ ${ }^{1,2,3}$ Department of electronics and communication engineering, GLA University, \\ Mathura, Uttar Pradesh, India \\ 1*pallavi.sr015@gmail.com, ${ }^{2}$ diwakar.agarwal@gla.ac.in, ${ }^{3}$ atul.bansal@gla.ac.in
}

\begin{abstract}
Biometric is the technology based on biological traits, which exploits the physical and behavioral characteristics of an individual. Ear biometric has gained immense attention over the last years. Because of its consistent shape and rich texture distribution, it is a reliable biometric for human recognition and identification. This paper presents an approach for ear based human identification using Wavelet transformation and Multi-scale Local Binary Pattern (MLBP). It exploits Haar wavelet decomposition up to fourth level and uniform texture distribution over the circular neighborhood region by varying the scale. Two different distance scores are incorporated for classification, namely, match distance and chi-square statistics. The proposed feature extraction and classification method are performed on IIT Delhi Ear Database, which has ear images acquired from 221 different subjects. The experimental results have shown better performance (in terms of accuracy) by an increment in a number of neighbors. The experimental results have shown better performance with the highest accuracy of $97.70 \%$ by an increment in a number of neighbors in MLBP, increasing the number of decomposition levels and also using different classifiers.
\end{abstract}

Keywords - Biometrics, Wavelet transform, Haar, MLBP, Feature vector, Match distance, Chi-square statistics

\section{INTRODUCTION}

The approach to scientifically identify humans came into existence in the 19th century when Alphonse Bertillon, biometric researcher, introduced law enforcement creating an identification system based on physical measurements. Biometrics is a biology-based systematic and technological authentication method, used in information assurance (IA). Using human biological information such as DNA or fingerprints, biometric identification validates reliable entry, data or access. The traits used in biometric should be behavioral or physiological in nature. Some examples of physiological biometric are a face, ear, iris, hand geometry, fingerprint, palm prints, hand veins geometry etc. and behavioral biometrics are voice, signature dynamics, motion recognition, keystroke dynamics etc. There are a number of systems that are developed which are based on these attributes and tested in real-world applications.

Among all the physiological characteristic, ear has emerged as reliable biometrics for human recognition and identification. In recent years, ear biometrics has become prominent due to [7]-

Received: May 10, 2019

Reviewed: August 12, 2019

Accepted: September 2, 2019 
1. The Ear is notably consistent in shape and also has invariable color distribution.

2. The shape of the ear does not change from 8 years to 70 years. Its appearance does not change with facial expressions.

3. Handling the background is not a tough job because the position of ear always remains fixed in the middle of the side face.

4. For capturing the ear images there is no need for much cooperation from us.

There are various research work in this particular field, some of which describe the background of the study are mentioned here. K. Jain et al. [8] presented biometric recognition framework, evolution, challenges, and future of biometric recognition over the last 50 years. Emeršic et al. [9] presented a review of the field of automatic ear recognition, also discussed challenges and potential research directions. Basit and Shoaib [24] presented an ear recognition technique constructed using curvelet transform. The feature extraction step is performed by applying fast discrete curvelet transform and for classification KMM is exploited. Anwar et al. [25] proposed an algorithm for geometrical features based on ear recognition. The pre-processed ear images are used by the snake model for detecting the ear.

Previous work done in the field of ear recognition proposed some very useful algorithms and techniques. But some of them give lower accuracy and few have longer computational time. This paper works towards the motivation to improve accuracy as well as time efficiency of the system by using improved feature sets and also different classifiers. This work contributes to the ear recognition field by giving two ear based human identification systems with an accuracy of $94.12 \%$ and $97.70 \%$, along the computational time (in seconds) of both the system. Also, equal error rates of the systems are calculated in order to make it possible for the user to decide in which region one needs to operate the system.

Section 1 and 2 of this paper includes introduction and literature survey of various ear recognition techniques respectively. Section 3 discusses the ear recognition system using Multiscale Local binary pattern and Section 4 discuss the ear recognition system using Wavelet transformation and Multiscale Local binary pattern. Section 5 presents a comparison of both the systems. And finally, Section 6 gives the conclusion of the work.

\section{LITERATURE SURVEY}

Several methods have been mentioned over the previous years for human ear recognition. Burge and Burger [1] proposed an ear biometric system which uses a graph matching algorithm. Ear images are modeled as adjacency graph obtained from the Voronoi diagram of a curved segment of the ear. Hurley et al. [2] presented a novel approach with an objective to reduce the dimensions on feature pattern space, but maintain the robust classification. They developed a system which treats the image as an array of Gaussian attractors which is the source of a force field, later directional properties of force fields are used to locate the extreme point of the potential. Choras [3] utilized geometrical features of ear such as shape, width, size, and height of ear lobe, which are very useful in identifying a human. Victor [4] proposed a Principle-based analysis (PCA) based face and ear biometric system using the same subjects. They used three different gallery combination for testing. Chang [5] presented an improved performance for face and ear biometric recognition system based on PCA using a larger dataset.

Kumar and Chan [6] proposed a method for ear recognition and verification in which ear representation is done using sparse coding based on Radon transformation. The solution is calculated by convex optimization technique. Prakash and Gupta [7] presented an ear recognition technique which deals with poor contrast and noise using image enhancement techniques. Then to minimize pose-variation SURF feature extraction technique is utilized. K. Jain et al. [8] presented biometric recognition framework, evolution, challenges, and 
future of biometric recognition over the last 50 years. Emeršic et al. [9] presented a review of the field of automatic ear recognition, also discussed challenges and potential research directions. Lei et al. [10] proposed an effective method of ear landmark localization, ear detection and pose classification. All three tasks are accomplished using the Ear treestructured graph (ETG).

Texture analysis has been an extensive research topic since the 1960s with potential areas of application including biomedical image analysis, document analysis, image coding, biometric person authentication etc. The different techniques that use texture analysis can be divided into four categories: statistical, geometrical, model-based and signal processing. The traditionally divergent structural and statistical models of texture analysis can be said to be integrated as Local binary pattern. Ojala et al. [11] proposed the design of an operator that can detect the Uniform Local Binary Pattern for different spatial resolutions, also they presented a method to integrate more than one operator for Multiresolution. Youbi et al. [12] presented the ear identification technique using the extension of LBP, which is called Multi-scale Local Binary Pattern, where the recognition rate is improved by varying the scale of the local circular neighborhood. Benzoic and Boukrouche [13] proposed a method to recognize human using only one sample of human ear image per person in the training set, they used the LBP texture operator for feature extraction. Support Vector Machine (SVM) is used as a classifier. Shan and Tommaso [14] propose to represent facial expressions using LBP and classifying the representation of face images using Support Vector Machine (SVM).

LBP codes can be collected in a histogram, but all histogram bins do not contain useful texture information. This method uses selective LBP histogram bins for facial representation. Hussain and Agarwal [15] proposed an algorithm which removes the artifacts (jaggies), from output images of LBP based edge detection technique, using Mathematical Morphological Interpolation in One step (mmINTone). This smoothens the edges of the flame. Wang et al. [16] proposed ear recognition method by combining the ULBPs (uniform local binary pattern) using the block-based and Multi-resolution methods on the sub-images of the ear which are transformed using Haar wavelet and classifying the images using Canberra distance. Kumar and $\mathrm{Wu}$ [17] proposed the approach that uses morphological operators and Fourier descriptors for segmenting the curved region of interest from the ear image. From feature extraction, they use localized orientation information and along with this, they examined local grey level phase information using complex Gabor filter. Zavar and Nixon [18] presented a model which proposed a part-wise description ear image obtained by random clustering on a group of scale invariable features of the training set. Further, they extended the model capturing information of ear's boundary structures using wavelet analysis.

Some of the latest work in this field are worth mentioning here. Emersic et al. [19] proposed an ear detection technique taking into account problems like low illumination and occlusions. For this purpose, they used convolution encoder-decoder networks (CEDs) which is based on SegNet architecture. Sarangi et al. [20] proposed a new ear recognition scheme which utilizes PHOG and LDA. Local features are extracted using pyramid histogram of oriented gradients (PHOG) and for dimension reduction of the PHOG descriptor, linear discriminant analysis (LDA) was used. The training and testing images are classified using nearest neighbour. Alqaralleh and Toygar [21] presented a 2D ear recognition method. Features are extracted from tragus and non-occluded part of the ear by local binary pattern (LBP) texture descriptor. Then the score between training and testing samples of the tragus and ear image are calculated separately. After this, the match scores of both tragus and ear image is fused and finally classified using KNN. A. Alshazly et al. [22] proposed an ear recognition approach based on gradient features., namely Histogram of Oriented Gradients (HOG), Local Optimal Oriented Patterns (LOOP), Local Directional Patterns (LDP), and Weber Local Descriptor (WLD). For classification chi-square similarity was incorporated. 
In this paper, an attempt has been made to propose an effective approach for ear based human identification based on a texture descriptor called Local Binary Pattern and its extension which is known as Multi-scale LBP (MLBP) along with Wavelet decomposition up to certain level. The proposed work has two approaches. In the first approach, the MLBP descriptor (Figure 1) based features are extracted from human ear images and the similarities between the pattern of the query image and the features extracted are captured using distance and then the decision is made using match distance. In the second approach, a combination of wavelet features and MLBP features are extracted from images and then similarities between the pattern of the query image and the features extracted are captured using Chi-square statistics. Results (in terms of accuracy) obtained for the second approach is better than the first one, as will be clear in the following sections.

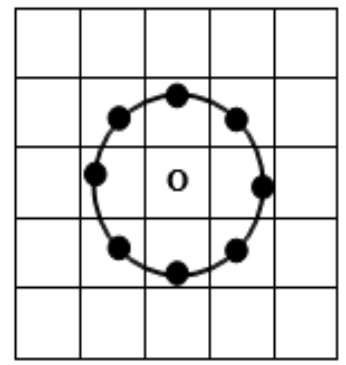

(b)

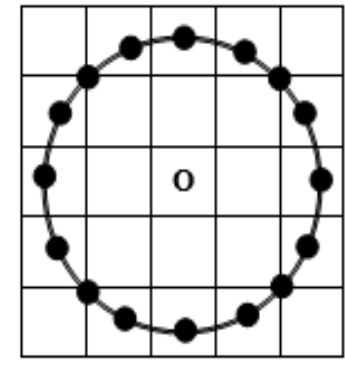

(c)

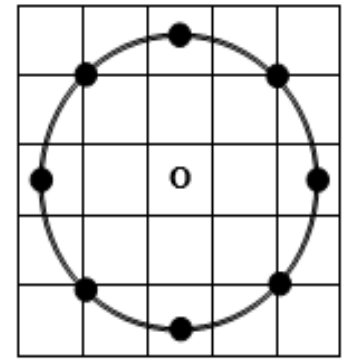

(a)

Fig. 1 The circular (a): $(8,1),(b):(16,2),(c):(8,2)$ Neighborhood

\section{PROPOSED SYSTEM USING MLBP}

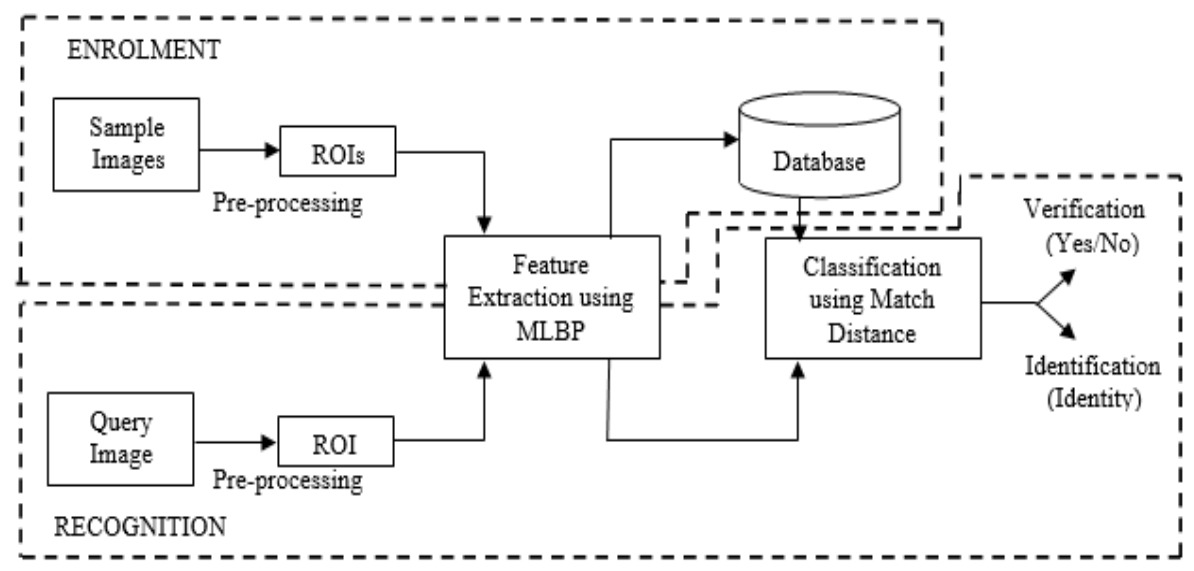

Fig. 2 Block diagram of Ear based human identification system using MLBP

Ear based human identification system is a combination of two steps, namely, enrolment and recognition. Figure 2 shows the block diagram of the ear based identification system using MLBP. In the enrolment step, ear images are first pre-processed and then using the MLBP descriptor the features are extracted, then feature vectors are accumulated in the database. In the second step, that is recognition, the query image is pre-processed and the feature pattern is extracted. These features are classified against the template stored in the database using match distance. After classification, the system decides that the image verified belongs to the desired person or not.

\subsection{FEATURE EXTRACTION USING MLBP}


Local binary pattern (LBP) is a texture operator analysis on a gray level image. LBP operator is used in real-world applications because of its invariant nature against its monotone changes in the grey level of the images. LBP was introduced by Ojala et al. [23]. The traditional local binary pattern works in a $3 \times 3$ neighborhood. LBP is calculated in a local circular region with $\mathrm{P}$ as the number of neighbors and $\mathrm{R}$ as the radius of the circular neighborhood (Figure 3). The center pixel value is subtracted from the other pixel present in the circular region. LBP operator marks the pixels of the image by thresholding each of the pixels of the local circular neighborhood with the value of the center pixel and summing up the products of threshold values and the weight matrix of the power of two. The LBP value for the center pixel is given by -

$$
L B P_{P, R}=\sum_{i=0}^{i-1} L B\left(p_{i}-p_{c}\right) \cdot 2^{i}
$$

Where,

$$
L B\left(p_{i}-p_{c}\right)= \begin{cases}1, & p_{i} \geq p_{c} \\ 0, & p_{i}<p_{c}\end{cases}
$$

$P=$ Number of a neighbor in the circular region

$R=$ The radius of the circular neighborhood

$p_{c}=$ Value of the central pixel of the circular neighborhood $p_{c} \in[0,255]$

$p_{i}=$ Value of other pixels of the circular neighborhood where $p_{i} \in[0,255]$

\begin{tabular}{|l|l|l|}
\hline 3 & 2 & 6 \\
\hline 5 & 5 & 6 \\
\hline 6 & 2 & 7 \\
\hline
\end{tabular}

(a)

\begin{tabular}{|l|l|l|}
\hline 0 & 0 & 1 \\
\hline 1 & & 1 \\
\hline 1 & 0 & 1 \\
\hline
\end{tabular}

(b)

\begin{tabular}{|c|c|c|}
\hline 1 & 2 & 4 \\
\hline 128 & & 8 \\
\hline 64 & 32 & 16 \\
\hline
\end{tabular}

(c)

Fig. 3 (a) $3 \times 3$ Window (b) Threshold Window $\left(\right.$ LBP Code $\left.=(00111011)_{2}=(59)_{10}\right)($ c $)$ Weight Window

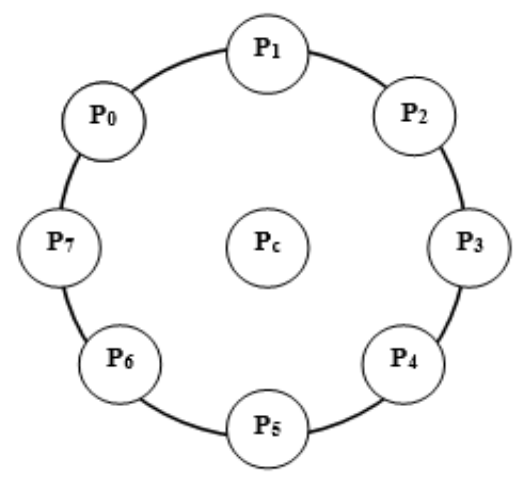

Fig. 4. LBP Features for an 8 Pixel Neighborhood 


$$
\begin{aligned}
L B P= & \left(p_{0}-p_{c}\right) * 2^{0}+\left(p_{1}-p_{c}\right) * 2^{1}+\left(p_{2}-p_{c}\right) * 2^{2}+ \\
& \left(p_{3}-p_{c}\right) * 2^{3}+\left(p_{4}-p_{c}\right) * 2^{4}+\left(p_{5}-p_{c}\right) * 2^{5}+ \\
& \left(p_{6}-p_{c}\right) * 2^{6}+\left(p_{7}-p_{c}\right) * 2^{7}
\end{aligned}
$$

The LBP codes of an image are accumulated into a histogram. The features extracted from the traditional LBP operator cannot capture the dominant texture elements. Therefore to subdue this limitation, in this work an extension of LBP is used, namely, Multi-scale local binary pattern (MLBP). In MLBP, radius $\mathrm{R}$ of the circular neighborhood is varied. In the proposed method, the idea is to split the image into an equal number of blocks and quantify MLBP on a single block at a time. The occurrence of MLBP codes is collected in the form of a histogram. The extracted MLBP features are integrated by concatenating the corresponding histogram by differing the values of radius $\mathrm{R}$ (Figure 5).

\subsection{CLASSIFICATION USING MATCH DISTANCE}

Extracted features of the query image are matched against the template stored in the database. A similarity score or distance checks whether the extracted feature belongs to the same ear image of a person or not. The classification used in the proposed work is matched distance. Match distance is a cumulative difference which measures dissimilarity between two probability distributions,

$$
D(H, K)=\sum_{i}\left|H_{i}-K_{i}\right|
$$

Where,

$$
\begin{aligned}
& H_{i}=\sum_{j \leq 1} H_{j}=\text { Cumulative Histogram of the query image } \\
& K_{i}=\sum_{j \leq 1} K_{j}=\text { Cumulative Histogram of the template stored in the database. }
\end{aligned}
$$
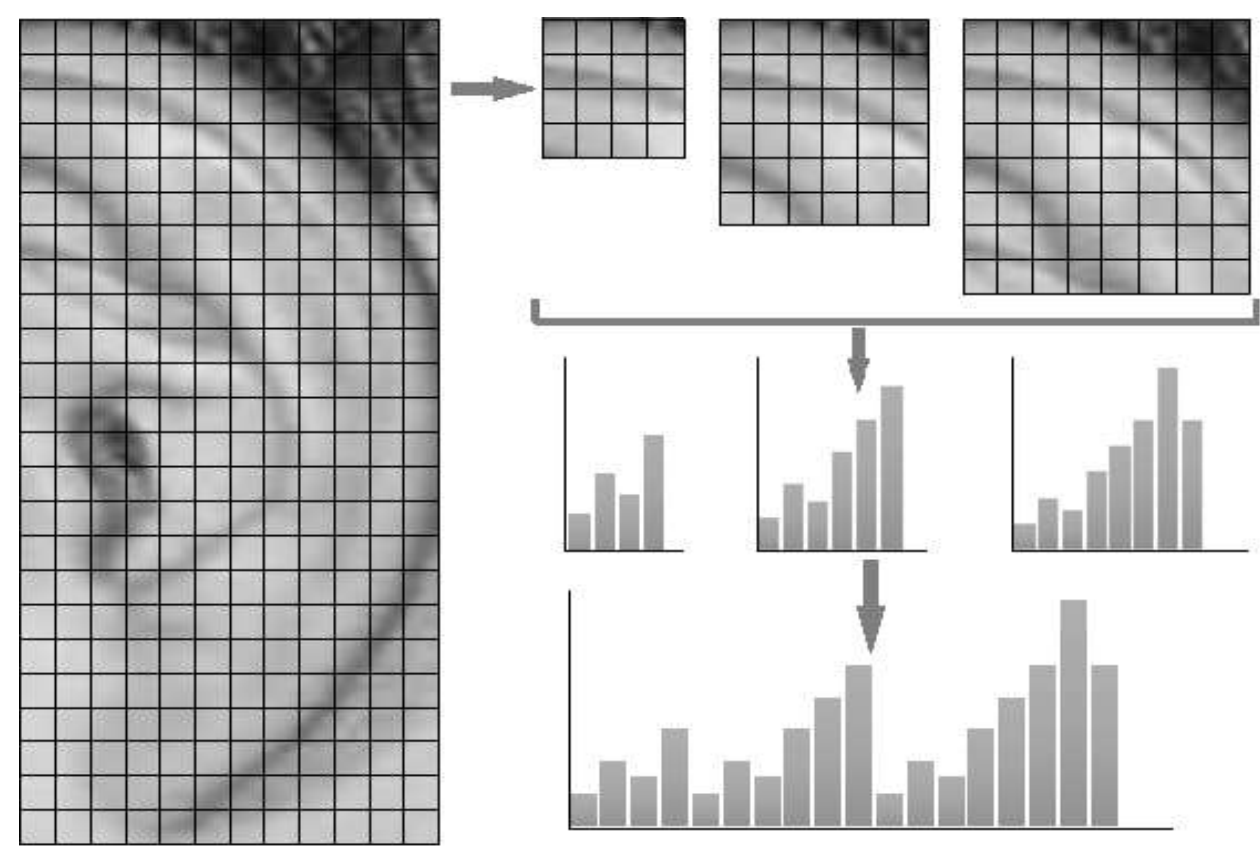
Fig. 5 Dividing the image window into blocks and concatenating the histogram

\subsection{RESULTS AND DISCUSSION}

3.3.1. DATABASE: The proposed system is conducted on IIT Delhi Ear Database. This database is acquired from 221 different subjects, where each subject has contributed at least three ear images. It makes a total of 754 ear images with resolution $272 \times 204$ pixels. Along with the original images, the database provides normalized and cropped ear images of size $50 \times 180$ pixels. The feature extraction technique is applied to these normalized and cropped ear images.

3.3.2. ANALYSIS OF THE RESULTS: After feature vector is extracted by applying MLBP descriptor the classification step uses Match distance to assess the performance of the feature extraction method. Table I shows the recognition results (in terms of accuracy) obtained on the previously mentioned ear database, in which 2 images per person are used for training and 1 sample image is used for testing. To compute MLBP with $\mathrm{P}=16$, three scales are used $R=\{2,3,4\}$. The best results here are obtained by splitting the image into 18 blocks. The performance of a biometric system is analyzed by computing different statistical measures like sensitivity, specificity, precision and ccuracy. Table II shows the analysis with different values of threshold on MLBP with $P=16$ and $R=\{2,3,4\}$. Dividing ear image into a number of blocks and then applying MLBP, gives better results in terms of accuracy rather than applying MLBP on the whole image at once.

Table I. Performance of the System with 2 training images and 1 test image

\begin{tabular}{|c|c|c|}
\hline No. of Neighbors & Radius & Accuracy(\%) \\
\hline $\mathbf{P}=\mathbf{8}$ & $\mathrm{R}=\{1,2,3,4\}$ & 82.8 \\
\hline $\mathbf{P}=\mathbf{1 6}$ & $\mathrm{R}=\{2,3,4\}$ & 94.12 \\
\hline
\end{tabular}

Table II. Statistical Performance Parameters with different values of threshold

\begin{tabular}{|c|c|c|c|c|}
\hline Threshold & Specificity & Sensitivity & Precision (\%) & Accuracy (\%) \\
\hline $\mathbf{0 . 2}$ & 0.91 & 0.96 & 94 & 94.12 \\
\hline $\mathbf{0 . 2 5}$ & 0.91 & 0.95 & 94 & 93.66 \\
\hline $\mathbf{0 . 3}$ & 0.92 & 0.93 & 94.6 & 92.76 \\
\hline $\mathbf{0 . 3 5}$ & 0.92 & 0.92 & 94.6 & 93.3 \\
\hline $\mathbf{0 . 4}$ & 0.96 & 0.909 & 97 & 91.8 \\
\hline $\mathbf{0 . 4 5}$ & 0.96 & 0.88 & 97 & \\
\hline
\end{tabular}

The false acceptance rate (FAR) shows the likelihood of ear based identification system that it will accept incorrectly the access of an ear image that is not in the database. The false rejection rate (FRR) shows the likelihood of the system that it will reject incorrectly the access of an ear image that is in the database. Figure.6 Shows the variation of FAR and FRR with respect to different values of the threshold. The value at which FAR and FRR 
curves meet is known as equal error rate (EER). EER is the rate at which FAR and FRR both the errors have equal values If this value of EER is low then the performance of the system is reliable. In this work value of EER is 0.0765 .

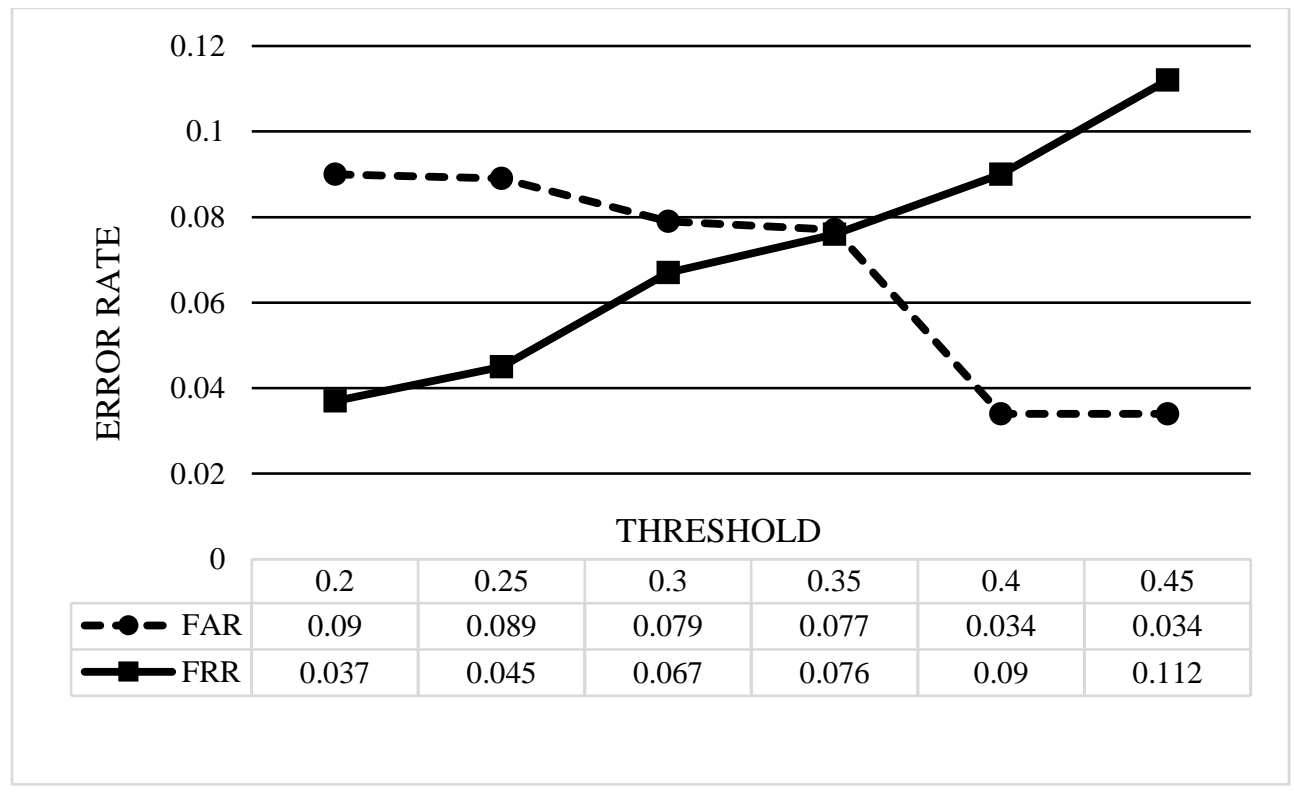

Fig. 6 Variation in FAR and FRR for different values of threshold

Moreover, Figure.6 let's decide that in which region system can be operated. If the system is designed such that the acceptation rate for unauthorized users can be high but the rejection rate for authorized users should be low then the system can be operated at 0.2 thresholds. If the system is designed such that the acceptation rate for the authorized user can be low but the rejection rate for the unauthorized user should be high then the system can be operated at a threshold value 0.45 . From this, it can be inferred that the feature extractor, i.e., MLBP, worked well when the radius is increased. Also, the execution of the classification step using match distance proves to work well with MLBP, as the identification in terms of accuracy is $94.12 \%$.

\section{PROPOSED SYSTEM USING HAAR WAVELET AND MLBP}

After obtaining performance parameters for the previously explained system, an attempt has been taken to explore new and improved feature set in focus to improving the performance parameters of the system. Figure 7 shows the model for ear based human identification using wavelet and MLBP features. In this model, first ear images are decomposed by Haar wavelet transform to obtain different component or combination of different components are split into an equal number of blocks in order to apply MLBP operator over one block at a time and repeated for all the blocks. The occurrence of MLBP codes for each block is collected in the form of histograms. And finally, these histograms are concatenated to form a single histogram for a component or combination of components (of a single image), which then serves as a feature vector.

\subsection{FEATURE EXTRACTION USING HAAR WAVELET AND MLBP}

Previously in the system described in Section 3, the ear image was being split up into an equal number of blocks and MLBP then applied on each block to collect the occurrence of MLBP codes in the form in form of a histogram. In this method, before dividing the image 
into blocks, wavelet transformation is applied to extract different components from the image. A wavelet transform is a mathematical tool that decomposes a signal, here an image, into a representation that gives detailed components. This representation can be used in applications like texture analysis, pattern recognition, noise reduction, data and image compression. Among the various wavelets, in this work, Haar wavelet is used. Haar wavelet is a series of rescaled functions which together form a wavelet family or basis. The haar sequence is known as the first wavelet basis.

\subsection{CLASSIFICATION USING CHI-SQUARE STATISTICS}

Finally for classification of textural features Chi-square statistics is used. Finally, a distance relationship is required to compare feature vectors of training and testing image. In Section 3, the match distance was used to calculate the dissimilarity between feature vectors of training and testing image. It is observed that when wavelet transformation is incorporated in the model, Chi-square statistics outperforms match distance.

$$
D(H, K)=\sum_{i} \frac{[f(i, H)-f(i)]}{f(i)}
$$

where,

$$
f(i)=\frac{f(i, H)+f(i, K)}{2}
$$

$H_{i}=$ Histogram of the Query Image

$K_{i}=$ Histogram of the template stored in the database

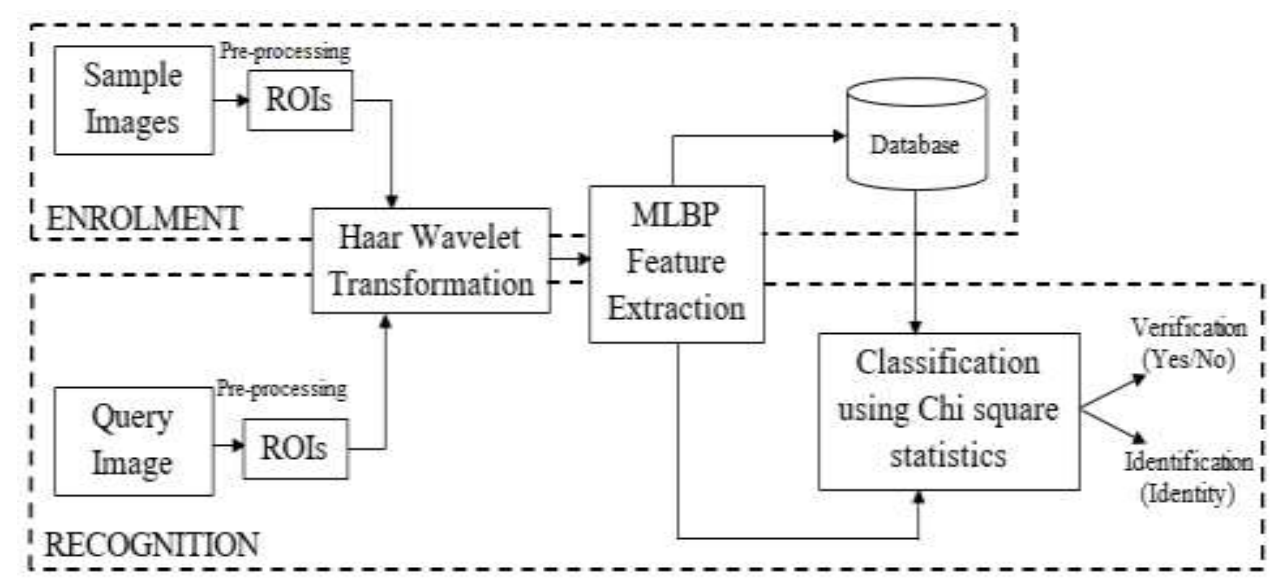

Fig. 7. Block Diagram of Ear Based Human Identification System using Wavelet transformation and MLBP

\subsection{RESULTS AND ANALYSIS}

After feature extraction using Haar wavelet and MLBP features, for classification match distance was incorporated. This classifier gave an accuracy of $85.00 \%$, which is lower than the previous system where the accuracy of $94.12 \%$. Also, the computational time calculated was 7.02 seconds. From the results, it was observed that the previous system gave better performance. So, to improve the performance of the system, chi-square statistics is used as a classifier. Classification step results in a similarity score, which is later used to calculate the performance parameters for this model. The database used here is the same as mentioned in Section 3.3.1, in which 2 images per person are used for training 
and 1 sample image is used for testing. The results are extracted up to the fourth level of wavelet decomposition. For MLBP descriptor number of neighbors for all the components and combination of components is $P=16$ for which three radius scales are used $R=\{2,3$, $4\}$.

The tables below show the performance parameters at the different level of decomposition. Performance parameters that are mention here are sensitivity, specificity, precision, and accuracy for approximation components and combinations of components at different levels. Table III shows the performance of the model at the first level, second level, third level and third level of wavelet decomposition. In the text ahead, LL refers to approximation component, LH refers to horizontal component, LV refers to vertical component of the first level; LL2 refers to approximation component, LH2 refers to horizontal component, LV2 refers to vertical component of the second level; LL3 refers to approximation component, LH3 refers to horizontal component, LV3 refers to vertical component of third level; LL4 refers to the approximation component, LH4 refers to horizontal component, LV4 refers to the vertical component of the fourth level.

It can be noticed form table III that the highest accuracy at first level is attained at a combination of approximation, horizontal and vertical components which is $94.10 \%$. Highest accuracy is attained at a combination of approximation, horizontal and vertical components which is $94.50 \%$ at the second level and $97.70 \%$ at third level. At the fourth level, the highest accuracy is obtained at a combination of approximation, horizontal and vertical components, i.e., $94.10 \%$.

Table III. Statistical performance of ear based human identification using a combination of wavelet and MLBP features at the different level of decomposition

\begin{tabular}{|c|c|c|c|c|}
\hline Feature Vector & Sensitivity & Specificity & $\begin{array}{c}\text { Precision } \\
(\%)\end{array}$ & $\begin{array}{c}\text { Accuracy } \\
(\%)\end{array}$ \\
\hline LL + MLBP & 0.97 & 0.86 & 91.40 & 92.70 \\
\hline LL + LH + MLBP & 0.98 & 0.86 & 90.60 & 93.20 \\
\hline LL + LV + MLBP & 0.97 & 0.87 & 91.30 & 93.20 \\
\hline LL + LH + LV + MLBP & 0.98 & 0.87 & 92.20 & $\mathbf{9 4 . 1 0}$ \\
\hline LL2 + MLBP & 0.97 & 0.87 & 91.30 & 93.20 \\
\hline LL2 + LH2 + MLBP & 0.98 & 0.88 & 92.80 & 94.10 \\
\hline LL 2 + LV2 + MLBP & 0.98 & 0.88 & 92.10 & 94.10 \\
\hline LL2 + LH2 + LV2 + MLBP & 0.98 & 0.89 & 93.40 & $\mathbf{9 4 . 5 0}$ \\
\hline LL3 + MLBP & 0.98 & 0.92 & 95.00 & 95.90 \\
\hline LL3 + LH3 + MLBP & 0.98 & 0.92 & 94.10 & 95.40 \\
\hline LL3 + LV3 + MLBP & 0.98 & 0.92 & 95.00 & 96.00 \\
\hline LL3 + LH3 + LV3 + MLBP & 0.98 & 0.96 & 97.80 & $\mathbf{9 7 . 7 0}$ \\
\hline LL4 + MLBP & 0.98 & 0.82 & 88.00 & 91.40 \\
\hline LL4 + LH4 + MLBP & 0.97 & 0.85 & 90.00 & 92.30 \\
\hline LL 4 + LV4 + MLBP & 0.97 & 0.86 & 90.50 & 92.70 \\
\hline LL4 LH4 + LV4 + MLBP & 0.97 & 0.90 & 92.70 & $\mathbf{9 4 . 1 0}$ \\
\hline & & & & \\
\hline & & & & \\
\hline
\end{tabular}


Similarly, it has been observed that system performance improves if we consider the combination of approximation (LL), horizontal (LH) and vertical (LV) components with a level of decomposition up to the third level. After which the accuracy starts falling. Also, it has been observed that features combining all three components give better performance as compared to single or two component and maximum accuracy of $97.7 \%$ is obtained at the third level with a combination of approximation component, a horizontal component, and a horizontal component.

Also, Table IV shows a different combination of approximation components of different levels is examined. When a combination of two approximation components is taken, the highest accuracy is obtained at a combination of LL2 and LL3 which is $95.90 \%$. Combination of LL1 and LL3 gives $95.40 \%$ accuracy which is a bit lower than the accuracy obtained from the combination of LL2 and LL3. Among all the combinations of three approximation components, the highest accuracy is acquired at a combination of LL1, LL2 and LL3 which is $94.10 \%$. While the combination of all the four approximation components, LL1, LL2, LL3 and LL4, gives the highest accuracy of $96.80 \%$. All these results are obtained using chi-square statistics in the classification step. Figure 8 shows the EER value for third level decomposition is 0.034 .

Table IV. Statistical Performance Parameters of the system for Combinations of Approximation Components.

\begin{tabular}{|c|c|c|c|c|}
\hline Feature vector & Sensitivity & Specificity & $\begin{array}{c}\text { Precision } \\
(\%)\end{array}$ & $\begin{array}{c}\text { Accuracy } \\
(\%)\end{array}$ \\
\hline LL1 + LL2 + MLBP & 0.98 & 0.89 & 93.00 & 94.10 \\
\hline LL1 + LL3 + MLBP & 0.98 & 0.92 & 95.80 & 95.40 \\
\hline LL1 + LL4 + MLBP & 0.94 & 0.92 & 94.70 & 93.20 \\
\hline LL2 + LL3 + MLBP & 0.98 & 0.92 & 95.00 & 95.90 \\
\hline LL2 + LL4 + MLBP & 0.98 & 0.88 & 92.90 & 93.60 \\
\hline LL3 + LL4 + MLBP & 0.97 & 0.89 & 92.70 & 93.60 \\
\hline LL1 + LL2 + LL3 + MLBP & 0.98 & 0.89 & 93.80 & 94.10 \\
\hline LL1 + LL2 + LL4 + MLBP & 0.94 & 0.92 & 94.70 & 93.20 \\
\hline LL2 + LL3 + LL4 + MLBP & 0.94 & 0.92 & 94.70 & 93.20 \\
\hline LL1 + LL2 +LL3 + LL4 + MLBP & 0.98 & 0.94 & 96.30 & $\mathbf{9 6 . 8 0}$ \\
\hline & & & & \\
\hline
\end{tabular}




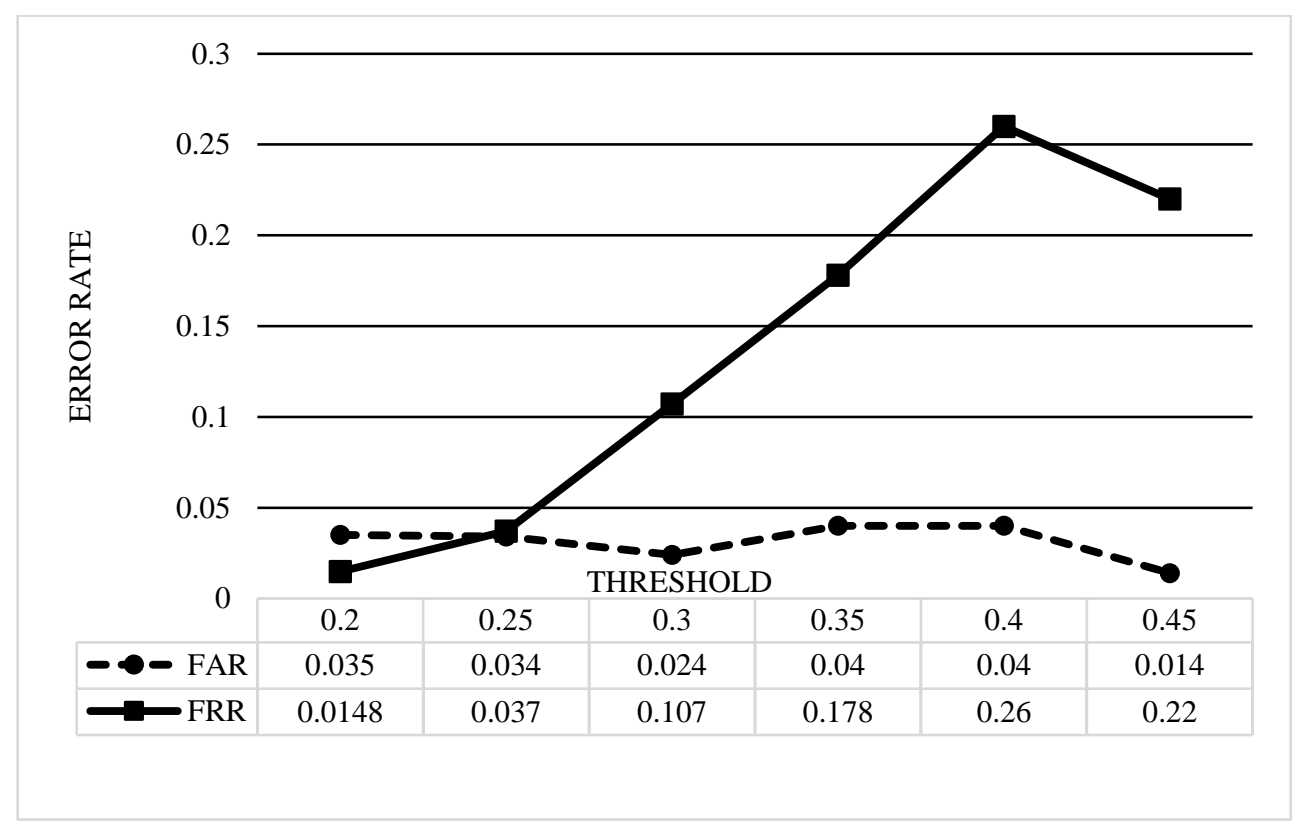

Fig. 8 Variation in FAR and FRR for different values of the threshold at the third level of decomposition

\section{COMPARISON OF BOTH THE APPROACHES}

In this paper, two ear recognition approaches are suggested, both performed on the same database, IIT Delhi Ear Database. In section 3, ear recognition based human identification system using MLBP is elaborated where first ear image is divided into an equal number of blocks and applying MLBP described over each block to later obtain histogram as a feature vector of each image. To compare the feature pattern of the Query image and feature vector of the template, a cumulative difference is used called, match distance. This approach reported accurately $94.12 \%$ with a computational time of 4.76 seconds.

In order to improve the performance of the system, an improved set is evolved using wavelet decomposition. In this second system (section 4), the ear image is first decomposed into different components using Haar wavelet. Then the selected components or combination of components are divided into an equal number of blocks. Later, MLBP descriptor is applied and the occurrence of the codes are obtained in the form of a histogram. In this system, a different classification is used called, Chi-square statistics.

The second approach outperforms the first approach with $97.70 \%$ accuracy, obtained at the third level of decomposition, with a computational time 6.07 seconds. In this approach, it has been observed as the decomposition level increases, the accuracy of the system also increases while considering approximation component (LL) only and maximum accuracy is obtained at third level, i.e., 95.9\%, after which accuracy starts decreasing. Similarly, it has been observed that system performance increases if we consider the combination of approximation (LL), horizontal (LH), vertical (LV) components with the level of decomposition up to the third level, after which accuracy start falling.

Also, the features combining all three components give better performance as compared to single or two components and maximum accuracy of $97.70 \%$ is obtained at the third level with a combination of LL3, LH3, and LV3. System performance has also been evaluated using a combination of approximation (LL) component of each level decomposition, it gives maximum accuracy of $96.8 \%$ combining LL1, LL2, LL3, and LL4. However, it is lesser than the combination of LL3, LH3, and LV3. Though the computational time of the second approach increases from the first approach, the accuracy of the second approach is higher than the first approach. 
Table V. Comparison of Ear recognition system using MLBP and Ear recognition system using Wavelet and MLBP

\begin{tabular}{|c|c|c|c|c|c|}
\hline & $\begin{array}{l}\text { Feature } \\
\text { Extraction }\end{array}$ & Classifier & $\begin{array}{c}\text { Precision } \\
(\%)\end{array}$ & $\begin{array}{c}\text { Accuracy } \\
(\%)\end{array}$ & $\begin{array}{c}\text { Computational } \\
\text { time } \\
\text { (seconds) }\end{array}$ \\
\hline $\begin{array}{c}\text { First } \\
\text { System }\end{array}$ & MLBP & Match distance & 94.00 & 94.12 & 4.76 \\
\hline \multirow{2}{*}{$\begin{array}{l}\text { Second } \\
\text { System }\end{array}$} & Haar + MLBP & Match distance & 87.00 & 85.00 & 7.02 \\
\hline & Haar + MLBP & $\begin{array}{l}\text { Chi square } \\
\text { statistics }\end{array}$ & 97.80 & 97.70 & 6.07 \\
\hline
\end{tabular}

Table VI. Comparison of performance of different feature vectors

\begin{tabular}{|c|l|l|c|c|}
\hline Feature Vector & Sensitivity & Specificity & $\begin{array}{c}\text { Precision } \\
(\boldsymbol{\%})\end{array}$ & $\begin{array}{c}\text { Accuracy } \\
(\boldsymbol{\%})\end{array}$ \\
\hline MLBP & 0.96 & 0.90 & 94.10 & 94.12 \\
\hline LL + LH + LV + MLBP & 0.98 & 0.87 & 92.20 & 94.10 \\
\hline LL2 + LH2 + LV2 + MLBP & 0.98 & 0.89 & 93.40 & 94.50 \\
\hline LL3 + LH3 + LV3 + MLBP & 0.98 & 0.96 & 97.80 & $\mathbf{9 7 . 7 0}$ \\
\hline LL4 + LH4 + LV4 + MLBP & 0.97 & 0.90 & 92.70 & 94.10 \\
\hline LL1 + LL2 + LL3 + LL4 + MLBP & 0.98 & 0.94 & 96.30 & 96.80 \\
\hline
\end{tabular}

On the basis of the explanation and table $\mathrm{V}$, the first is a little faster than the second approach, while maintaining higher accuracy proves to perform better than the second system. But among the second approach, the results obtained using Chi-square statistics are better than the results obtained using match distance. Table VI and Figure 9 elaborate performance of both the systems with different feature vectors. It shows the highest accuracies with each feature vector. Performance of the system using Haar wavelet features and MLBP features at a different level of decomposition gives different accuracy. The system with MLBP as feature extractor and match distance as classifier give the highest accuracy of $94.12 \%$ at threshold 0.2 .

Among all the different feature vectors combining Haar wavelet and MLBP with Chisquare statistics as a classifier, a combination of LL3, LH3, LV3, and MLBP gives the highest accuracy of $97.7 \%$ at threshold 0.2 . Feature vector combining approximation component of the four decomposition levels and MLBP descriptor feature, i.e., LL1, LL2, 
LL3, LL4, and MLBP gives an accuracy of $96.8 \%$, which is the second highest among all feature vectors.

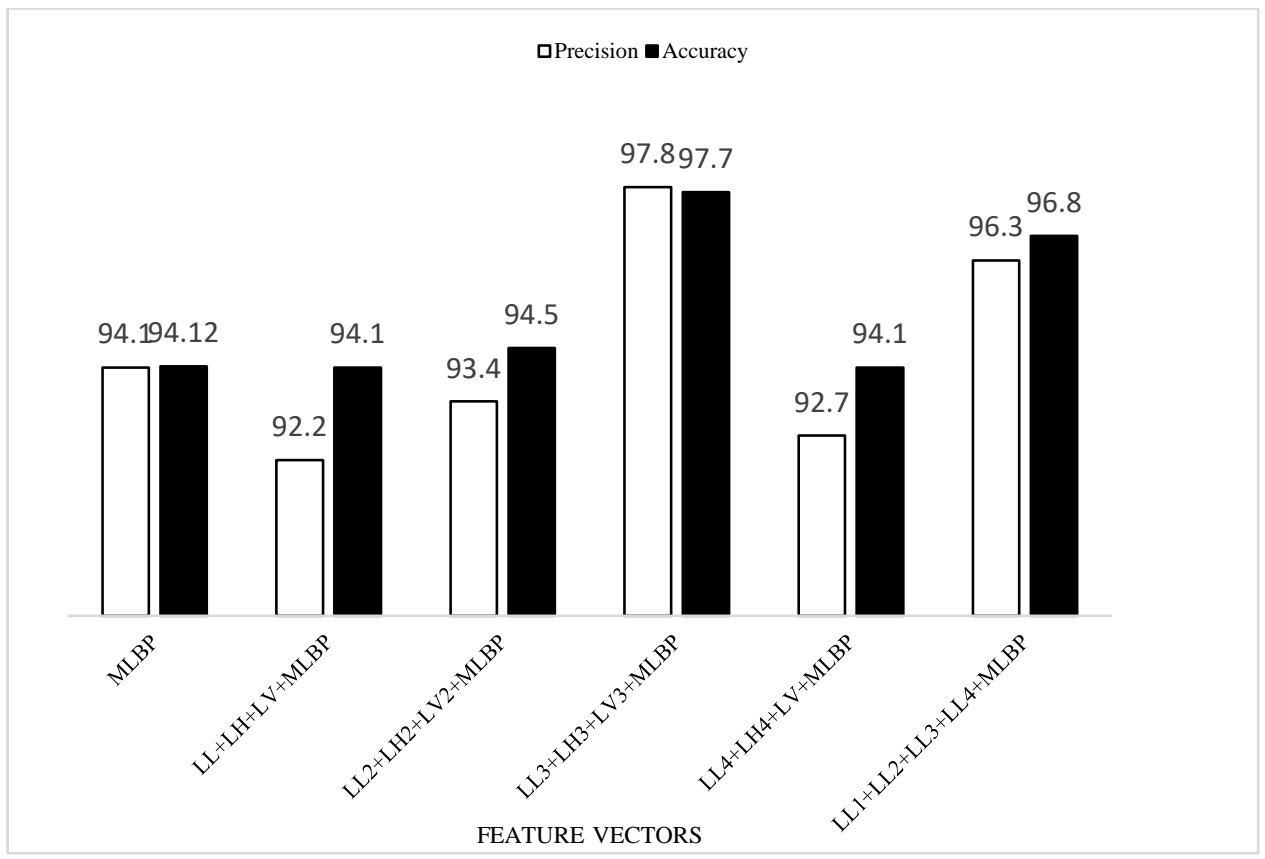

Fig. 9 The accuracy of the system with different Feature Vectors

\section{CONCLUSION}

In this work, two ear based human identification systems are proposed, a first system using only texture descriptor MLBP and other using Wavelet transform and MLBP, with classification performed by Match distance and Chi-square statistics respectively, to obtain a dissimilarity score between feature vector of the training set and the feature pattern. The results are obtained after performing the experiment on IIT Delhi ear database. Where first system reported recognition rate of 94.12 with computational time 4.76 seconds. And second system reported recognition result of highest accuracy $97.70 \%$ and computation time 6.07 seconds among all the combinations of feature vectors. The performance can further be improved using different classification techniques. It has been observed that as the decomposition level increases, the accuracy of the system also increases. While considering only LL component, maximum accuracy is acquired at the third level, i.e., 95.90\%. Combining all the four approximation component, LL1, LL2, LL3, and LL4, gives an accuracy of $96.80 \%$. A comparison between both the systems is also shown. It can be said that as the number of neighbors in MLBP increases, the accuracy of the system also increases. Further, this work can be extended using different classification techniques, and also by considering improved feature set.

\section{REFERENCES}

[1] Burge, M. and Burger, W., 2000. "Ear biometrics in computer vision". Proceedings of the 15th International Conference on Pattern Recognition, Barcelona, Spain.

[2] Hurley, D.J., Nixon, M.S. and Carter, J.N., 2002. "Force field energy functionals for image feature extraction". Image and Vision comput. 20, 311-317.

[3] Choraś, M., 2008. "Perspective methods of human identification: ear biometrics". Opto-electronics review.16,85-96.

[4] Victor, B., Bowyer, K. and Sarkar, S., 2002. "An evaluation of face and ear biometrics". Proceedings of Object Recognition Supported by user Interaction for Service Robots, Quebec, Canada. 
[5] Chang, K., Bowyer, K.W., Sarkar, S., and Victor, B., 2003. "Comparison and combination of ear and face images in appearance-based biometrics". IEEE Transactions on pattern analysis and machine intelligence. 25, 1160-1165.

[6] Kumar, A. and Chan, T.S.T., 2013. "Robust ear identification using a sparse representation of local texture descriptors". Pattern recognition, 46, 73-85.

[7] Prakash, S. and Gupta, P., 2013. "An efficient ear recognition technique is invariant to illumination and pose". Telecommunication Systems, 52,1435-1448.

[8] Jain, A.K., Nandakumar, K. and Ross, A., 2016. "50 years of biometric research: Accomplishments, challenges, and opportunities". Pattern Recognition Letters, 79, 80-105.

[9] Emeršič, Ž., Štruc, V. and Peer, P., 2017. "Ear recognition: More than a survey”. Neurocomputing, 255 , 26-39.

[10] Lei, J., You, X. and Abdel-Mottaleb, M., 2016. “Automatic ear landmark localization, segmentation, and pose classification in range of images". IEEE Transactions on Systems, Man, and Cybernetics: Systems, 46,165-176.

[11] Ojala, T., Pietikainen, M. and Maenpaa, T., 2002. "Multiresolution gray-scale and rotation invariant texture classification with local binary patterns". IEEE Transactions on pattern analysis and machine intelligence, 24,971-987.

[12] Youbi, Z., Boubchir, L., Bounneche, M.D., Ali-Chérif, A. and Boukrouche, A., 2016. "Human ear recognition based on multi-scale local binary pattern descriptor and KL divergence". Proceedings of 39th International Conference on Telecommunications and Signal Processing, Vienna, Austria.

[13] Benzaoui, A. and Boukrouche, A., 2017. "Ear recognition using local color texture descriptors from one sample image per person". Proceedings of 4th International Conference on Control, Decision and Information Technologies, Barcelona, Spain.

[14] Shan, C. and Gritti, T., 2008. "Learning Discriminative LBP-Histogram Bins for Facial Expression Recognition". Proceedings of The British Machine Vision Conference, Leeds, U.K.

[15] Hussain, Z. and Agarwal, D., 2015. "Artifacts removal in LBP based flame edges using binary morphology". Proceedings of International Conference on Signal Processing, Computing and Control, Waknaghat, India.

[16] Wang, Y., Mu, Z.C. and Zeng, H., 2008. "Block-based and Multi-resolution Methods for Ear Recognition Using Wavelet Transform and Uniform Local Binary Patterns". Proceedings of $19^{\text {th }}$ International Conference on Pattern Recognition, Tampa, USA.

[17] Kumar, A. and Wu, C., 2012. "Automated human identification using ear imaging". Pattern Recognition, 45, 956-968.

[18] Arbab-Zavar, B. and Nixon, M.S., 2011. "On guided model-based analysis for ear biometrics. Computer Vision and Image Understanding"', 115, 487-502.

[19] Emeršič, Ž., Gabriel, L.L., Štruc, V. and Peer, P., 2017. Pixel-wise ear detection with convolutional encoder-decoder networks. arXiv preprint arXiv:1702.00307(2017).

[20] Sarangi, P.P., Mishra, B.S.P. and Dehuri, S., 2017 . "Ear recognition using pyramid histogram of orientation gradients". 4th International Conference on Signal Processing and Integrated Networks, IEEE, Noida, India ,590-595.

[21] Alqaralleh, E. and Toygar, Ö., 2018. "Ear recognition based on fusion of ear and tragus under different challenges. International Journal of Pattern Recognition and Artificial Intelligence,32,p.1856009.

[22] Alshazly, H.A., Hassaballah, M., Ahmed, M. and Ali, A.A., 2018. "Ear biometric recognition using gradient-based feature descriptors". International Conference on Advanced Intelligent Systems and Informatics, Springer, Cairo, Egypt, 435-445.

[23] Ojala, T., Pietikainen, M., and Harwood, D., 1994. Performance evaluation of texture measures with classification based on Kullback discrimination of distributions". Proceedings of 12th International Conference on Pattern Recognition, Jerusalem, Israel.

[24] Basit, A., and M. Shoaib., 2014, "A human ear recognition method using nonlinear curvelet feature subspace". International Journal of Computer Mathematics, 91, 616-624.

[25] Anwar, Asmaa Sabet, Kareem Kamal A. Ghany, and Hesham Elmahdy., 2015, "Human ear recognition using geometrical features extraction”. Procedia Computer Science, 65, 529-537. 
International Journal of Future Generation Communication and Networking Vol. 12, No. 3 (2019) 